\title{
تأثير معدلات تسميد نيتروجيني على بعض الخصائص الفسيولوجية لمحصول الشعير بمنطقتي مراوة والوسيطة بالجبل الأخضر في ليبيا. (Hordeum vulgare L.)
}

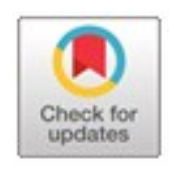

\author{
الطيب فرج حسين *، أحمد عبد الله بوكليلة \\ كلبة الزراعة، قسم المحاصبل-جامعة عدر المختار، البيضاء - ليبيا.
}

تاريخ الاستلام: 28 أكتوبر 2018 / تاريخ القبول: 30 يونيو 2019 https://doi.org/10.54172/mjsc.v34i2.86:Doi

المستخلص: تهدف هذه الدراسة لمعرفة سلوك محصول الثعير باختلاف البيئة لمستويات النيتروجين المضاف في صورة يوريا

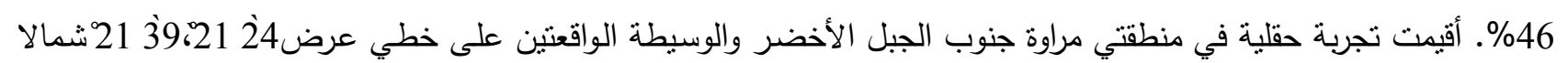
و32 20، 4732 شرقاً وترتفعان 508 و 185 متراً فوق مستوى سطح البحر للمنطقتين بالترتيب خـلال الموسم الزراعي 2014

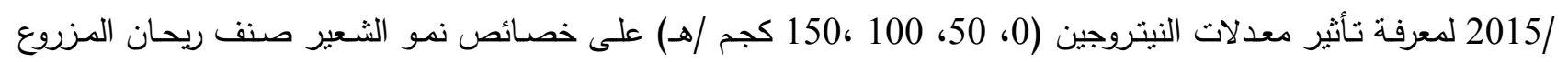
بالمعدل 70 كجم/هـ بالتسطير على مسافة 15سم. صممت تجربة القطاعات كاملة العشوائية في 4 مكررات في وحدات تجريبية

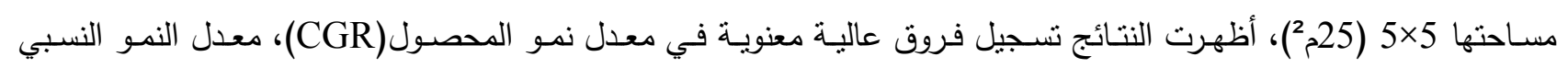

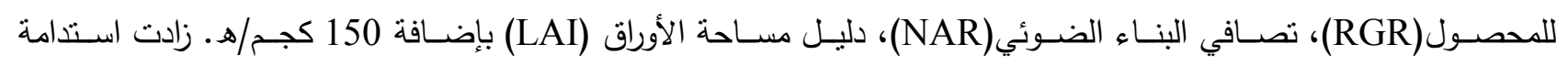

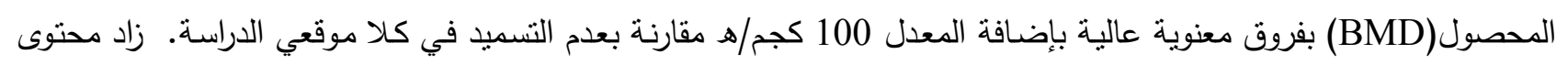

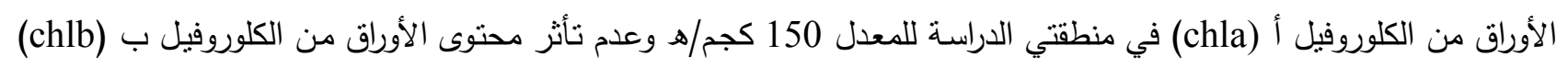
والكلي (Tchl) في مراوة وبفروق معنوية في الوسيطة عند إضافة 150 كجم/هـ لكلوروفيل ب و 100 كجم/هـ للكلوروفيل الكلي.

الكلمات المفتاحية: الثعير، السلوك الفسيولوجي، معدلات التسميد النيتروجيني

العالم (جمعية المهندسين الزراعيين الكوينيـة، 2016) وتقدر

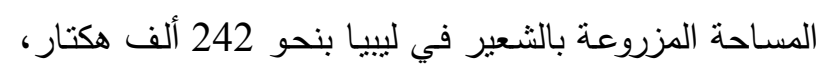
منها نحو 58 ألف هكتار تحت النظام المرويّ بمعدل إنتاج بـاج 2.89 طن/هـ والباقي تحت النظام المطري بمعدل إنتاج 0.91

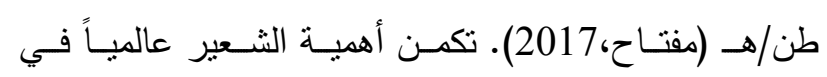

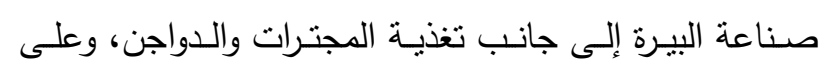

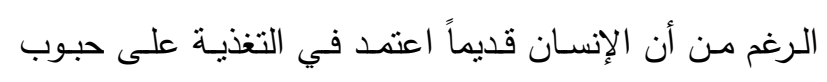

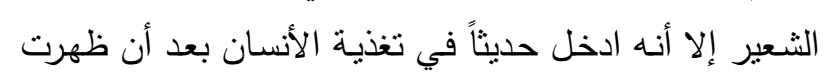

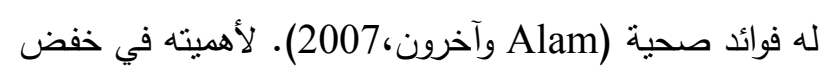

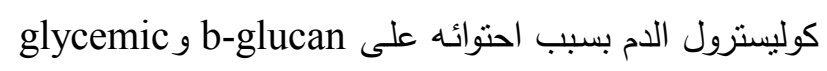

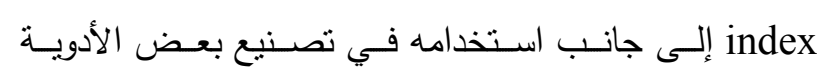

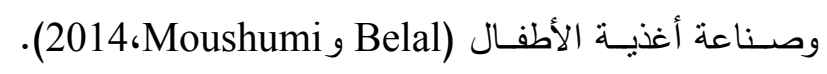
وبما أن المعروف عن الثعير أنه يتصف بانخفاض حاجته الثال

\section{المقدمة}

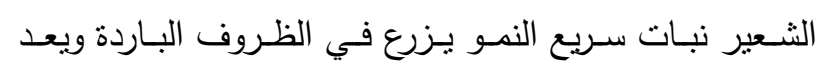

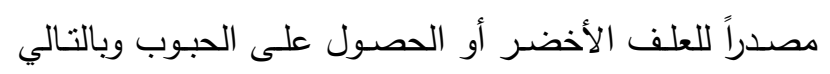

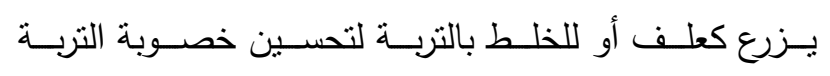
Ghanbari) المرتبة الخامسة ضمن محاصيل الحبوب في العالم بعد الذرة

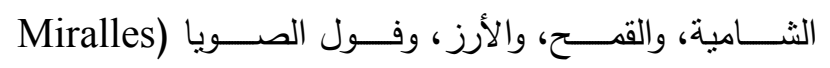

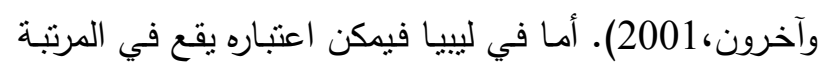

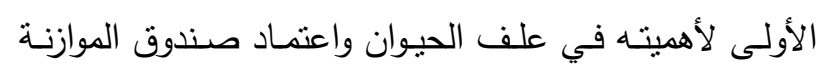

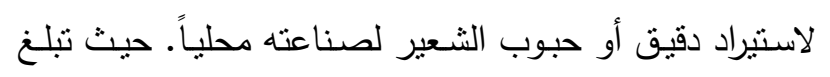
المسـاحة العالميـة للثعير 54059705 مليون هكتار وإنتـاج حبوب 8225383 مليون طن، وتعد كل من روسيا وأوكرانيا

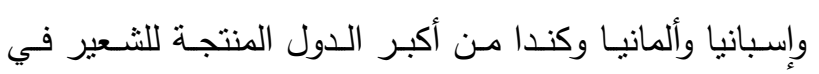


50سمفي مرحلة الأشطاء لتقدير الوزن الجاف الثاني. قبل التجفيف ثم قياس المساحة الورقية للمحصول خلال مرحلة 4 أوراق (LA1) ومرحلة التتطئة (LA2) بحيث قدرت المساحة باستخدام: طـول الورقـة ل عـرض الورقـة × 0.75 كمـا أثنــار

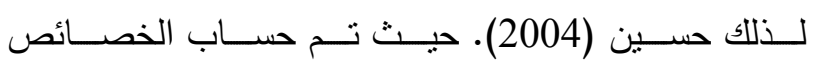
الفسيولوجية على النحو الآتي: معدل نمو المحصول (CGR)

$$
\mathrm{CGR}=\frac{1}{S A}(\mathrm{~W} 2-\mathrm{W} 1) /(\mathrm{T} 2-\mathrm{T} 1)
$$

حيث W2 ، W2 الوزن الجاف لوحدة المسـاحة خـلال الزمن الأولT1 والثاني T2 المساحة الأرضية المشغولة معدل النمو النسبي للمحصول(T) (T) (T) RGR= InW2-InW1/ T2-T1 معدل استدامة المحصول $(\mathrm{BMD}) \mathrm{BMD}=\quad(\mathrm{W} 2+\mathrm{W} 1 / 2) \quad(\mathrm{T} 2-\mathrm{T} 1)$

تصافي البناء الضوئي (NAR) $\mathrm{NAR}=(\mathrm{W} 2-\mathrm{W} 1 / \mathrm{T} 2-\mathrm{T} 1) \quad(\mathrm{InLA} 2-\mathrm{InLA} 1 / \mathrm{LA} 2-$ LA1) حيث LA1، LA2 المساحة الورقية في الزمن الأول والثاني للقياس دليل مساحة الأولق (LAI) عند بداية الطرد $\mathrm{LAI}=\mathrm{LA} / \mathrm{SA}$

محتــوى الأورلق مــن الكـــورفيلات (أ، ب والكـــي) وذلــك

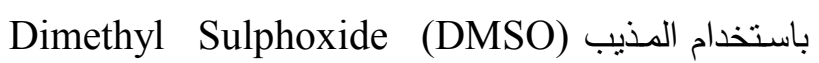

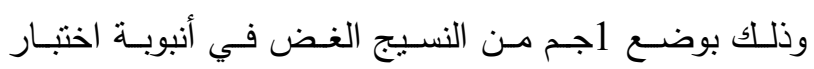

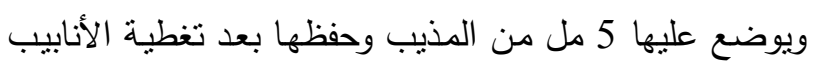
بورق السلوفان في الظلام في الثناجة لمدة 24 ساعة ثم يرشح

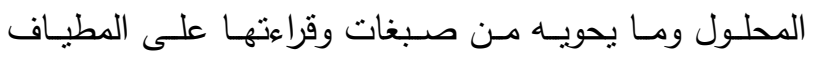

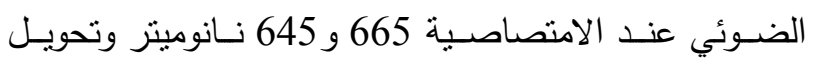
الامتصاصية إلى تركيز مجم/جم بالاستعانة بالمعادلات Chl (a) $=11.75 \times \mathrm{A} 665-2.35 \times \mathrm{A} 645 \times \frac{1}{v o l}$
للماء مقارنة بالقمح والأرز والذرة والصويا ولذلك تنجح زراعة

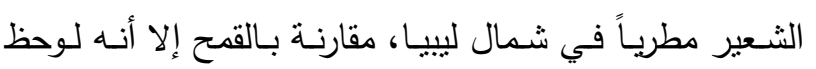

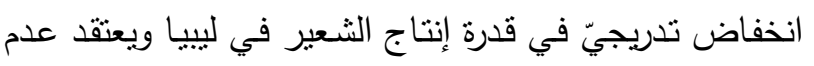

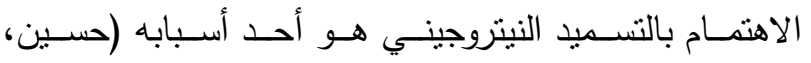
2005). من المعروف أَنَّ الثُعير حساس للتسميد النيتروجيني

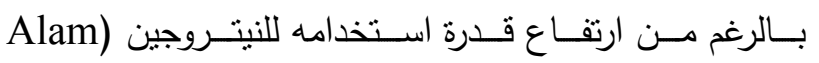

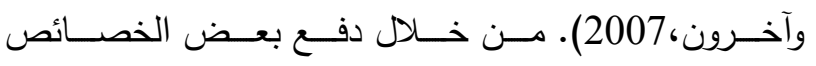

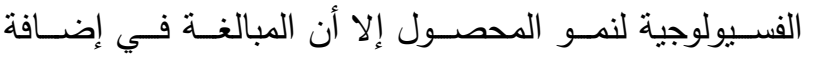

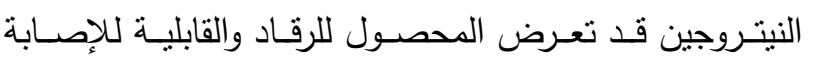

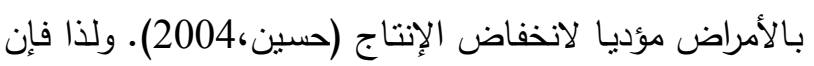

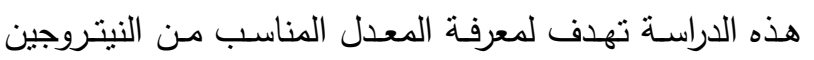

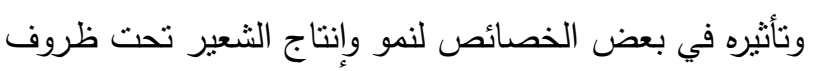

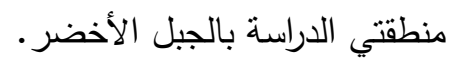

\section{المواد وطرق البحث}

أقيمت تجربة حقلية في منطقتي مراوة جنوب الجبل الأخضر

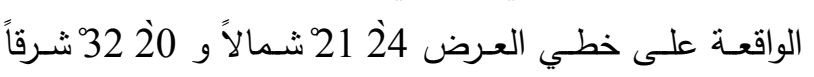
بارتفاع 508 منر فوق سطح البحر والوسيطة الواقعة عند 21 39 شمالاً 47 ند شرقاً وترتفع 185 متراً فوق سطح البحر

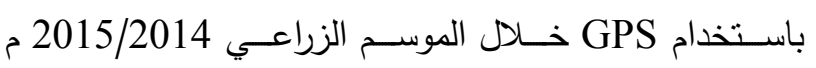

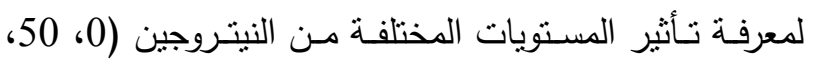

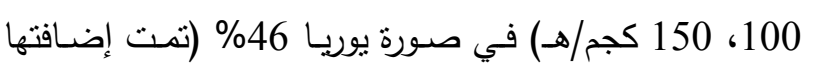

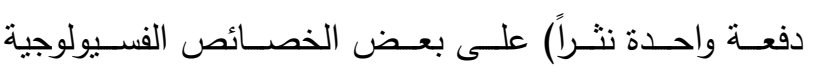

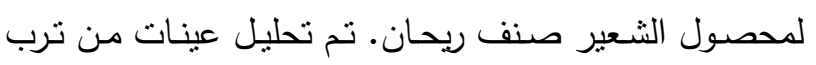

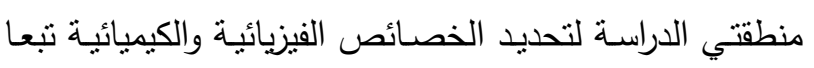
(1965،Black)

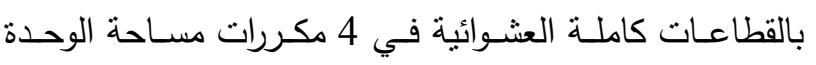
التجريبية 25م² والزراعة بمعدل 70كجم/هـ بالتسطير المسافة بينها 15سـم في منتصف شـهر نـوفمبر • تم حصـاد مسـاحة

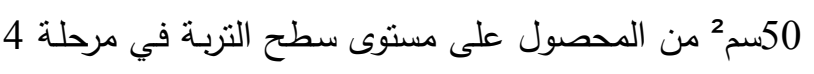
أوراق (35 يوماً من الإنبات) وتجفيفها في المجفف على درجنة

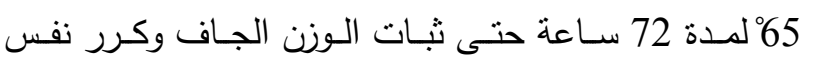

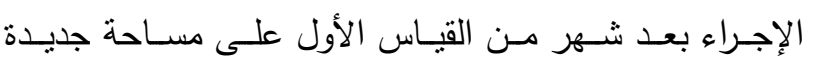




\section{النتائج والمناقشة}

معدل نمـو المحصـول (CGR) جـ/2²/ /يـوم: سجلت فروق

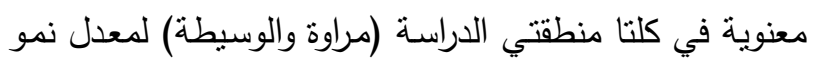
المحصول باختلاف مستويات النيتروجين المضاف كان معدل

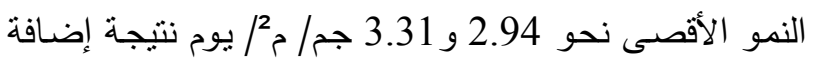
المعدل 150 كجم نيتروجين/هـ وعند النظر لأقل معدل نمو النو تهو

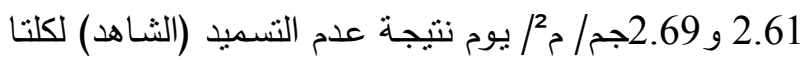

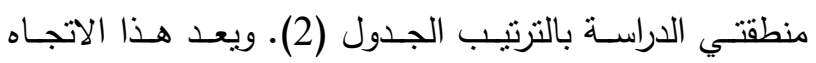

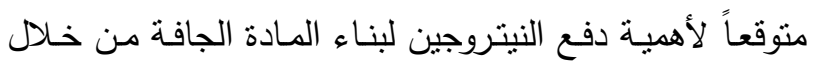

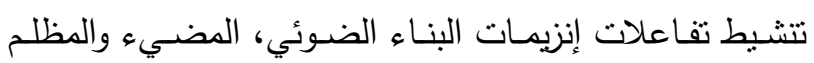
إلى جانب تحفيز البروتين الوظيفي أو التخزيني، بالتالي ينتهي

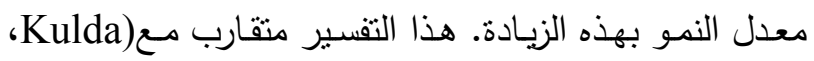

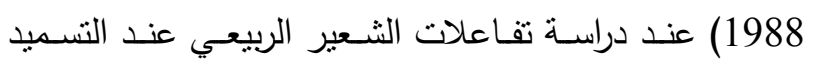

\section{معدل النمـو النسبي (RGR): جـ/ جم مسادة جافة/ يـوم:}

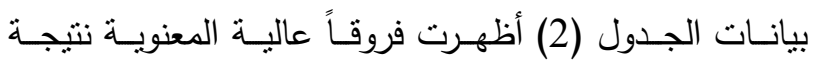
معدلات النيتروجين المضاف أدناه عند الثـاهد (عدم التشميد)

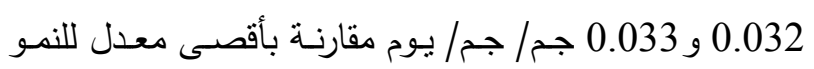
النسبي 0.036 و 0.040 جم/ جم/ يوم نتيجة التسميد بالمعدل

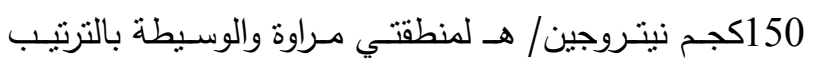

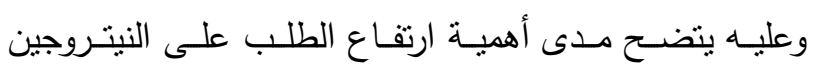

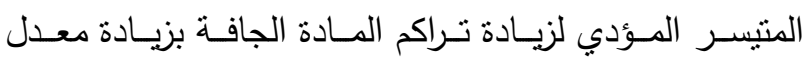

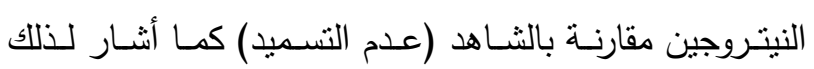

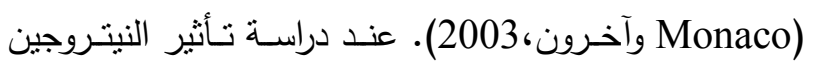
على سرعة الإنبات ونمو البادرات.

معدل استـامة المحصول (BMD) جم/ م²/ يـوم: أظهرت

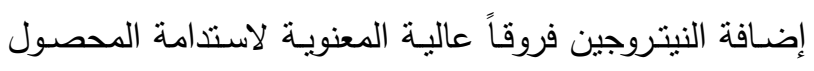

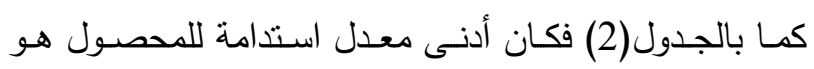
3.04 و 4.11 جم/ م²/ يوم في حالة الثـاهد (عدم التسمبد)

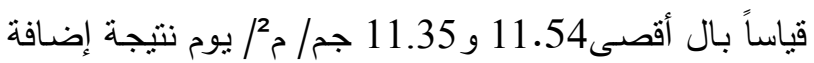
النيتروجين بالمعدل 100كجم نيتروجين/ هـ لمنطقتي مـراوة
Chl (b) $=18.61 \times \mathrm{A} 645-3.96 \times \mathrm{A} 665 \overline{\frac{1}{v o l}} \times$ Total chl $=7.04 \times \mathrm{A} 665-20.27 \times \mathrm{A} 645 \times \frac{1}{v o l}$ حيث vol من حجم المذيب المستخدم. وذلك كما أشـار لذلك (Rupaha وآخرون،2010). الجدول(1). الخصائص الفيزيائية والكيميائية لنرب منطقتي مراوة والوسيطة

\begin{tabular}{|c|c|c|}
\hline \multicolumn{3}{|c|}{ الخصائص الفيزيائية * } \\
\hline الوسيطة & مراوة & \\
\hline 48.15 & 23.81 & رمل \% \\
\hline 18.52 & 38.09 & طين \% \\
\hline 33.33 & 38.10 & سلت \% \\
\hline طي & طيني طمي & القوام \% \\
\hline \multicolumn{3}{|c|}{ الخصائص الكيميائية } \\
\hline 7.8 & 8.1 & $\mathrm{pH}$ \\
\hline 0.19 & 0.34 & $\left(\mathrm{dsm}^{-1}\right) \mathrm{EC}$ \\
\hline 4.7 & 4.8 & السعة التبادلية (ملي \\
\hline & & مكافئ/لتر ) \\
\hline 0.89 & 0.82 & مادة عضوية \% \\
\hline 0.08 & 0.11 & نيتروجين كلي \% \\
\hline 0.97 & 1.42 & $\% \mathrm{Na}^{+}$ \\
\hline 0.15 & 0.11 & $\% \mathrm{Mg}^{+2}$ \\
\hline 0.09 & 0.15 & $\% \mathrm{Ca}^{+2}$ \\
\hline 6 & 9 & $(\mathrm{ppm}) \mathrm{K}^{+}$ \\
\hline 10.03 & 15.56 & $\% \mathrm{Co}_{3}{ }^{-2}$ \\
\hline 0.21 & 0.11 & $\% \mathrm{CL}^{-}$ \\
\hline 0.38 & 0.70 & $\% \mathrm{Hco}_{3}^{-}$ \\
\hline
\end{tabular}

"قللت الترب بمعمل الأراضي بقسم التربة، كلية الزراعة، جامعة عمر المختار

التحليـل الإحصـائي: تخضـع جميع البيانـات المجعـة لتحليل

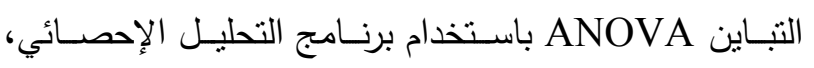
النسخة المعدل 9.2 (2009) لاختبار تأثنير المعاملات

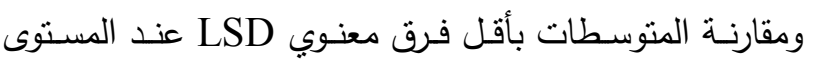

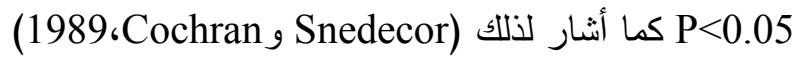


النبتروجين في صفة تراكم المادة الجافة لبادرات الثعبير . دليـل مسـاحة الأولق ( LAI ) عنــ بلايـة الطرد: أثنـارت بيانات الجدول(2) إلى فروق معنوية عالية في دليل مساحة

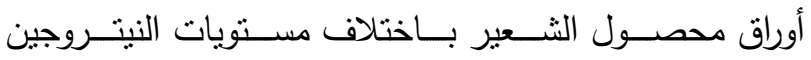
المضـاف في كلتا منطقتي الدراسـة الأصـغر 4.96 و 4.64

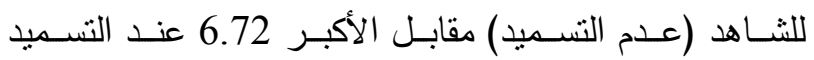
بالمعـدل 150 كجـم نيتروجين/هـ لمنطقتـي الدراســة (مـراوة

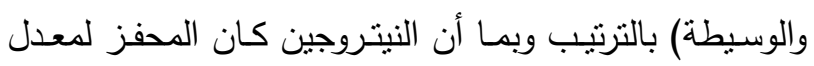
نمو المحصول وتصافي البناء الضوئي واستدامة المحصول فإن المحصلة هو تكوين مساحة ورقية مثالية بالنسبة لمساحة الأرض المشغولة حيث وصلت إلى ما يقارب اعتراض 95 بونة

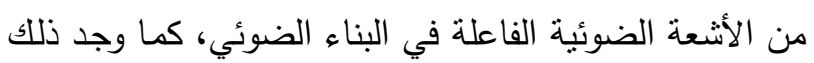
(Mahboubeh) في الشكل الظاهري للشعير •
والوسيطة بالترتيب، ونتير هذه النتائج لأهمية النيتروجين في

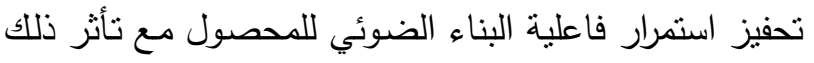

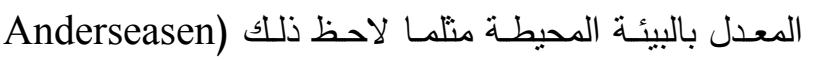

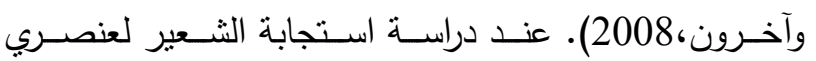
النيتروجين والفوسفور •

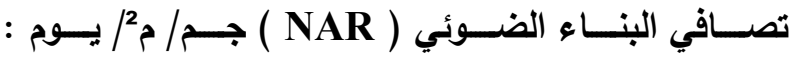

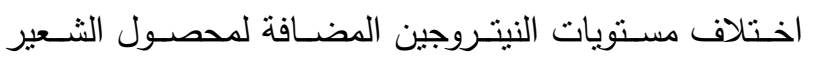

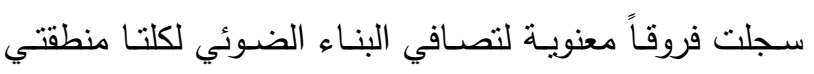

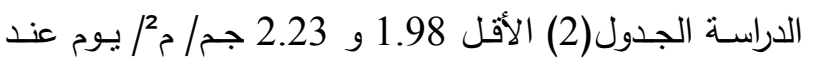

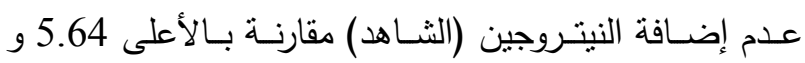

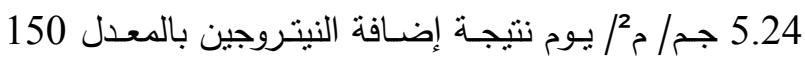

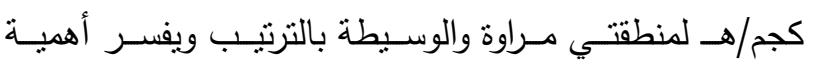
النيتـروجين للمحافظـة على مكونـات وفاعليـة وظيفــة البنـاء

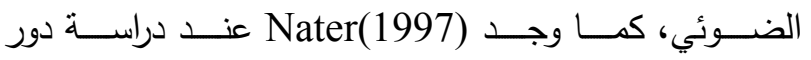

الجدول(2). تأثير مسنويات النيتروجين المضاف على خصائص نمو محصول الثُعير لمنطقتي مراوة والوسيطة بالجبل الأخضر

\begin{tabular}{ccccccccccc}
\hline \hline & \\
\hline
\end{tabular}

وبنفس استجابة كلوروفيل ب استجاب المحتوى من الكلوروفيل

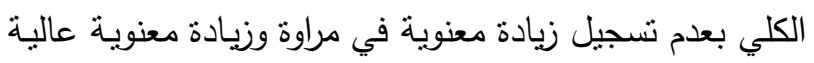
في الوسيطة الاقصى 0.107 عند إضافة النيتروجين بالمعدل فراليتل

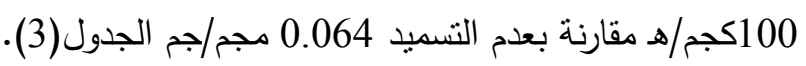
ولما كان كلوروفيل أ هو أسـاس التفاعلات الكيموضوئية لذا لذانيا فهو يعادل تقريبا ضـف المحتوى من كلوروفيل ب ويتجلى دور النيتروجين في بناء مكونـات الكلوروفيل، وقد تعزى هذه هنه النتيجة إلى أن تربـة مراوة البور كان محتواهـا من النيتروجين

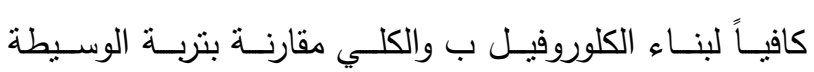

محتوى الأوراق من الكلورفيلات ( أ، ب و الكلي ) مجم/ جم نسيج غض : زيادة معدل النيتروجين المضـاف حتى 100 كجم أدت إلى زيادة عالية المعنوية لمحتوى الأوراق من صبغة معلة كلوروفيل أ 0.046 و 0.047 مجم/ جم مقارنة بعدم التسميد 0.032 و 0.031 مجم/جم لمنطقتي مراوة والوسيطة بالترتيب

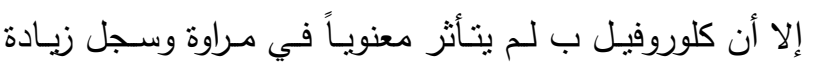

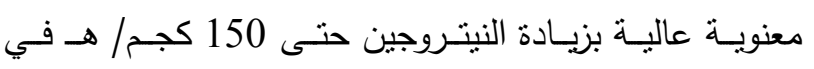

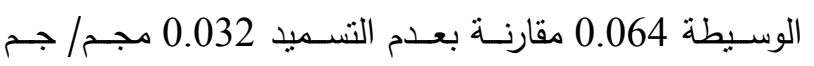




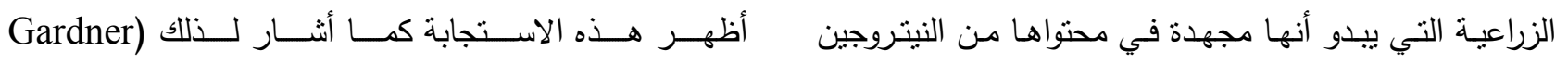

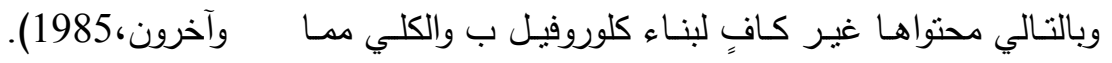

الجدول(3). تأثير مستويات النيتروجين المضاف (كجم/ه) على محتوى أوراق محصول الثعبير من الكلورفيلات مجم/جم نسيج غض لمنطقتي مراوة والوسيطة بالجبل الأخضر

\begin{tabular}{|c|c|c|c|c|c|c|}
\hline \multicolumn{2}{|c|}{ الكلوروفيل الكلي } & \multicolumn{2}{|c|}{ كلورفيل ب } & \multicolumn{2}{|c|}{ كلوروفيل أ } & \multirow{2}{*}{ النيتروجين كجم/ هـ الكلوفيلات } \\
\hline الوسيطة & مراوة & الوسبطة & مراوة & الوسيطة & مراوة & \\
\hline $0.064 \mathrm{c}$ & 0.064 & $0.0320 \mathrm{~d}$ & 0.0321 & $0.031 \mathrm{~d}$ & $0.032 \mathrm{~d}$ & 0 \\
\hline $0.099 \mathrm{~b}$ & 0.141 & $0.0550 \mathrm{c}$ & 0.0963 & $0.043 b$ & $0.044 \mathrm{~b}$ & 50 \\
\hline $0.107 \mathrm{a}$ & 0.107 & $0.0600 \mathrm{~b}$ & 0.0615 & $0.047 \mathrm{a}$ & $0.046 \mathrm{a}$ & 100 \\
\hline $0.106 \mathrm{a}$ & 0.108 & $0.0640 \mathrm{a}$ & 0.0649 & $0.041 \mathrm{c}$ & $0.043 \mathrm{c}$ & 150 \\
\hline$* *$ & N.S & $* *$ & N.S & $* *$ & $* *$ & $\mathrm{~F}$ \\
\hline 0.002 & - & 0.001 & - & 0.0030 & 0.0016 & LSD \\
\hline
\end{tabular}

(d، c، b،a) يشير إلى الفروق بين المتوسطات عند مستوى معنوية 0.05 العمود المكون من الحروف

مفتاح، فرج خميس. (2017). تأثنير إضـافة مخلفات الكرنب

الطـازج ومسـتويات النيتـروجين علـى نمـو وإنتـاج

\section{الخلاصة}

الثـعير والحشــائش المصـاحبة تحـت ظـروف مـراوة

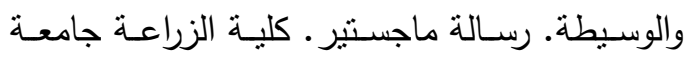

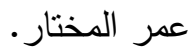

في الختام يوصي الباحث بأن إضافة النيتروجين بالمعدل

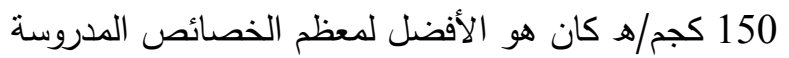
في كلا موقعي الدراسة.
Alam, M., Haider, S., \& Paul, N. (2007). Yield and yield components of barley (Hordeum vulgare L.) cultivars in relation to nitrogen fertilizer. Journal of Applied Sciences Research, 3(10), 1022-1026.

Andreasen, C., Litz, A. S., \& Streibig, J. C. (2006). Growth response of six weed species and spring barley (Hordeum vulgare) to increasing levels of nitrogen and phosphorus. Weed research, 46(6), 503-512.

Black, C. A. (1965). Methods of soil analysis, part II. Amer. Soc. Inc. Pub., Madison, USA, 770-779.

Dhawan, R. S., Bhasker, P., Chawla, S., Punia, S., Singh, S., \& Angrish, R. (2010). Impact of aryloxyphenoxypropionate herbicides on Phalaris minor in

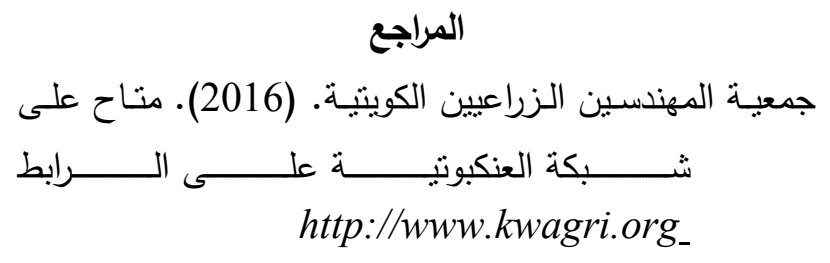

حسين، الطيب فرج. (2004). استجابة محصـول الثعير (1) صـنف الكـوف (Hordeum vulgareL.) لمعـدلات ودفعـات إضــافة النيتــروجين. المجلـــة المصـرية للعلـوم التطبيقيـة، مجلـد 19 عـد (10): $.155-126$

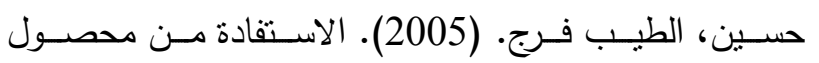

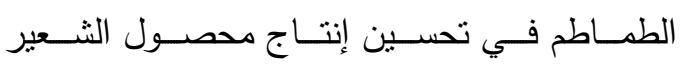
(Hordeum vulgare .L)

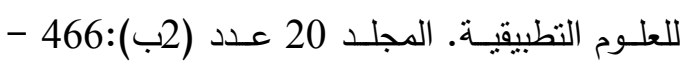
.477 
Mousavi, M., Soleymani, A., \& Shams, M. (2012). Effect of cultivars and nitrogen on growth and morphological traits of barley in Isfahan region. International Journal of Agriculture and Crop Sciences (IJACS), 4(22), 1641-1643.

Natr, L. (1997). The effect of nitrogen on dry matter allocation in young spring barley plants (Hordeum vulgare). Scientia Agriculturae Bohemica (Czech Republic).

Snedecor, G. W., \& Cochran, W. G. (1989). Statistical methods, 8thEdn. Ames: Iowa State Univ. Press Iowa.
Haryana. Indian Journal of Weed Science, 42(3and4), 136-143.

Gardner, F.P ; Earce, R.B.P and Michelle, R.L.(1985). Physiology of crop plants (ed). Iowa state Univ. press Arwes, pp:327

Ghanbari, A., Babaeian, M., Esmaeilian, Y., Tavassoli, A., \& Asgharzade, A. (2012). The effect of cattle manure and chemical fertilizer on yield and yield component of barley (Hordeum vulgare). African Journal of Agricultural Research, 7(3), 504-508.

Hossain, B., \& Akhtar, M. (2014). Growth and yield of barley (Hordeum vulgare L.) as affected by irrigation, sowing method and phosphorus level. Academia Journal of Agricultural Research, 2(1), 30-35.

Institute, S. (2009). Using JMP Student Edition for Windows and Macintosh: The User's Guide to Statistics with JMP Student Edition: SAS Institute.

Kulda, M. (1988). Reaction of spring barley lines to increased nitrogen fertilization. Field. Crop. Abst , 2(9), 1990

Miralles, D. J., Ferro, B. C., \& Slafer, G. A. (2001). Developmental responses to sowing date in wheat, barley and rapeseed. Field Crops Research, 71(3), 211-223.

Monaco, T. A., MacKown, C. T., Johnson, D. A., Jones, T. A., Norton, J. M., Norton, J. B., \& Redinbaugh, M. G. (2003). Nitrogen effects on seed germination and seedling growth. Rangeland Ecology \& Management/Journal of Range Management Archives, 56(6), 646-653. 


\title{
Effect of Nitrogen Fertilization Rates on Some Physiological Properties of Barley Crop (Hordeum vulgare L.) in Marwa and Al-Wasitah Regions in Al- Jabal Al-Akhdar, Libya.
}

\author{
Tayeeb Farag Hessain*, Ahmed Abdallah Bukalela \\ Faculty of Agriculture, Agronomy Department, Omar Al-Mukhtar University, Libya
}

Received: 28 October 2018/ Accepted: 30 June 2019

Doi: https://doi.org/10.54172/mjsc.v34i2.86

\begin{abstract}
The aim of this study is to investigate the barley crop (Hordeum vulgare L.) behavior in different environments to the levels of nitrogen added in the form of urea $46 \%$. A field experiment was conducted in the areas of Marawah, south of Al-Jabal Al-Akhdar, and Wesata which are located at 2124,2139 North , 32 20,32 47 East, and 508, $185 \mathrm{~m}$ above sea level for both sites respectively, to study the effect of nitrogen levels $(0,50,100$ and 150 $\mathrm{kg} \mathrm{ha}^{-1}$ ) on some of the growth traits of barley( c.v Rehan) sown in the rate of $70 \mathrm{~kg} \mathrm{ha}^{-1}$ by drilling (15cm apart) during the growing season 2014/15. The experiment was laied out in RCBD by 4 replicates with $25 \mathrm{~m}^{2}$ for each. The results showed significant differences in crop growth rate (CGR), relative growth rate (RGR), photodegradation (NAR), leaf area index (LAI) with $150 \mathrm{~kg} / \mathrm{h}$. Sustainable yield (BMD) significantly increased with the addition of $100 \mathrm{~kg} / \mathrm{h}$ compared to non-fertilization in both study sites. leaves chlorophyll a , b and total content responsed to nitrogen levels by significant increase $(\mathrm{P}<0.01)$ in chl a in both sites and at Wesataincase of chl $b$ and total, meanwhile chl $b$ and total were not affected by nitrogen levels in Marawa.
\end{abstract}

Keywords: Barley. Physiological behavior. Nitrogen levels. 